2013-10-15

\title{
Humidity and Temperature Effect on Properties of Transmission Gratings Recorded in PVA/AA-Based Photopolymer Layers
}

Tatsiana Mikulchyk

Technological University Dublin, Tatsiana.mikulchyk@tudublin.ie

Suzanne Martin

Technological University Dublin, suzanne.martin@tudublin.ie

Izabela Naydenova

Technological University Dublin, izabela.naydenova@tudublin.ie

Follow this and additional works at: https://arrow.tudublin.ie/scschphyart

Part of the Optics Commons

\section{Recommended Citation}

Tatsiana Mikulchyk, Suzanne Martin, Izabela Naydenova, Humidity and temperature effect on properties of transmission gratings recorded in PVA/AA-based photopolymer layers. 2013 J. Opt. 15105301. doi:10.1088/2040-8978/15/10/105301

This Article is brought to you for free and open access by the School of Physics \& Clinical \& Optometric Science at ARROW@TU Dublin. It has been accepted for inclusion in Articles by an authorized administrator of ARROW@TU Dublin. For more information, please contact arrow.admin@tudublin.ie, aisling.coyne@tudublin.ie, gerard.connolly@tudublin.ie.

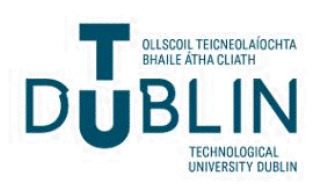




\title{
Humidity and temperature effect on properties of transmission gratings recorded in PVA/AA-based photopolymer layers
}

\author{
Tatsiana Mikulchyk, Suzanne Martin and Izabela Naydenova* \\ Centre for Industrial and Engineering Optics/School of Physics, College of Sciences and \\ Health, Dublin Institute of Technology, Kevin Street, Dublin 8, Ireland \\ E-mail: izabela.naydenova@dit.ie
}

\begin{abstract}
This paper explores the effects of humidity on gratings recorded in a PolyvinylalcoholAcrylamide photopolymer medium. Investigation of the behaviour of transmission gratings exposed to high humidity is of significant interest for two reasons, firstly because the grating's sensitivity to humidity can be exploited for the development of irreversible humidity indicators, secondly because too much sensitivity to humidity can limit the use of these materials in applications where an environmentally stable hologram is needed. In this paper we focus on the effect of high humidity on the properties of volume phase transmission gratings recorded in PVA/AA photopolymer layers in the temperature range of $8-24{ }^{0} \mathrm{C}$. It has been found that although exposure to humidity changes the diffraction efficiency and Bragg angle of gratings, the effects are fully reversible if the temperatures are kept low. For example, when gratings were subjected to relative humidity of $80 \%$ and $90 \%$ at a temperature of $8{ }^{0} \mathrm{C}$ the observed changes were fully reversible. However, irreversible changes in diffraction efficiency, thickness, refractive index modulation and Bragg angle were observed when the temperature during the humidity exposure was higher than $16{ }^{0} \mathrm{C}$. The magnitude of the irreversible changes depends strongly on the ambient temperature during the humidity exposure, the humidity level and also on the duration of the humidity exposure.
\end{abstract}

Keywords: holographic recording materials; photopolymers; acrylamide based photopolymer; volume holographic gratings; relative humidity.

Subject classification numbers: $42.70 \mathrm{Ln}, 42.40 \mathrm{~Pa}, 42.40 . \mathrm{Eq}, 42.70 . \mathrm{Gi}$ 


\section{Introduction}

Acrylamide-based photopolymer is under continuous study because of its possible practical applications, such as holographic interferometry, holographic optical elements, holographic data storage and holographic sensors [1-10]. This material has received much attention due to its wide dynamic range, high sensitivity, low scattering, self-processing nature and relatively low cost. Acrylamide-based photopolymer most commonly consists of monomers acrylamide and $\mathrm{N}, \mathrm{N}$ methylenebisacrylamide, photosensitizer, photoinitiator triethanolamine and the binder polyvinylalcohol. However, due to the highly hygroscopic nature of the polyvinylalcohol binder and the polyacrylamide produced during the recording of the hologram, the sensitivity of this photopolymer film to humidity can be significant. It has often been observed that in certain environmental conditions the diffraction efficiency, Bragg angle of recorded gratings, and even the layer surface can be affected by moisture or humidity. Often, the effects are fully reversible and the hologram regains its original properties when the humidity returns to normal, as described below. However, in some circumstances high humidity has been observed to cause irreversible changes to the recorded hologram, even in transmission gratings. Research is needed to fully characterise the response of transmission gratings to humidity changes and to better understand the nature of the irreversible changes that sometimes occur. This knowledge could allow development of irreversible humidity indicators to provide warning of high humidity exposure, and may also help in the development of more stable photopolymer materials for applications where an environmentally stable hologram is essential, such as holographic data storage or holographic optical elements. Thus, in order to achieve the full potential of acrylamide-based photopolymer, it is required to understand its behaviour at different levels of relative humidity $(\mathrm{RH})$ and know the moisture stability limit.

The poor stability of some acrylamide-based photopolymer films with triethanolamine as an electron donor has been reported recently $\left(\mathrm{RH}=80 \%\right.$ and $\left.\mathrm{T}=25{ }^{\circ} \mathrm{C}\right)$ [11]. To decrease the sensitivity to humidity the authors proposed $\mathrm{N}$-phenylglycine as an initiator. In [8] the humidity response of a volume reflection hologram recorded in an acrylamide-based photopolymer has been investigated and used to develop a visual indicator of environmental humidity in the humidity range of $5-80 \%$. The reflection hologram changes its colour when exposed to different levels of humidity due to the changed fringe spacing as the medium gains or loses moisture. This change in the fringe spacing is fully reversible. Later, responses of a volume reflection hologram to humidity $(10-80 \%)$ and temperature $\left(15-50{ }^{\circ} \mathrm{C}\right)$ were studied in more detail [12]. In that case the humidity sensitivity of reflection gratings recorded in an acrylamide-based photopolymer was investigated at $\mathrm{RH}<80 \%$ and only reversible change of holographic grating properties were observed. Research into the effects of humidity on transmission gratings is very limited and has been carried out only at $\mathrm{RH}=60 \%$ [13]. It has been shown that after exposure to humidity of $60 \%$ the diffraction efficiency of transmission grating reaches its initial value measured at low humidity of $20 \%$.

In this paper we analyse the behaviour of diffraction efficiency, thickness, refractive index modulation and Bragg angle of the volume transmission gratings recorded in acrylamide-based photopolymer layers under humidity exposure at different temperatures. Specifically we aim to discuss the irreversible changes of the properties of transmission gratings caused by exposure to high humidity $(\mathrm{RH} \geq 80 \%)$ and the role of temperature on these changes.

\section{Theory}

In order to record a volume phase holographic grating, coherent light from the laser is split to form an object beam and a reference beam. A transmission volume phase grating can be produced when the photosensitive medium is placed in the region of overlap of these two beams which reach the photosensitive medium from the same side. The interference pattern induced by two light waves is recorded as a spatial modulation of the refractive index of the photosensitive medium. Volume phase grating regime corresponds to $Q>>1$, which is defined by the relation [14]: 


$$
Q=\frac{2 \pi \lambda d}{n \Lambda^{2}}
$$

where $\lambda$ is the wavelength of the recoding light, $d$ is the thickness of the grating, $n$ is the average refractive index of the medium, $\Lambda$ is the fringe spacing.

In this study, transmission gratings with spatial frequency of approximately 1000 lines/mm have been recorded in the photosensitive layers with the thickness of $80 \pm 5 \mu \mathrm{m}$. These parameters correspond to a $Q$ factor of about 200 and, thus, allow us to apply the coupled wave theory [15] for the calculation of the refractive index modulation $(\Delta n)$. According to the coupled wave theory, $\Delta n$ is determined by:

$$
\Delta n=\frac{\lambda \cos \theta_{B} \sin ^{-1}(\sqrt{\eta})}{\pi d},
$$

where $\lambda$ is the wavelength of the reconstructing beam, $\theta_{B}$ is the Bragg angle inside the photopolymer layer at this wavelength, $\eta$ is diffraction efficiency of the recorded grating. $\theta_{B}$ is related to the external Bragg angle $\left(\theta_{B}\right)$ by Snell's law:

$$
n \sin \theta_{B}=\sin \theta_{B}^{\prime}
$$

$\theta_{B}{ }^{\prime}$ is given by Bragg's law:

$$
2 \Lambda \sin \theta_{B}^{\prime}=\lambda
$$

where $\lambda$ and $\Lambda$ are defined as in eq.(1).

\section{Experimental}

A self-processing acrylamide-based photopolymer developed at the Centre for Industrial and Engineering Optics, Dublin Institute of Technology [16-18] was used as a holographic recording material. The photosensitive solution optimized for recording in transmission mode consisted of two monomers - $0.6 \mathrm{~g}$ acrylamide and $0.2 \mathrm{~g} \mathrm{~N}, \mathrm{~N}$-methylene bisacrylamide, $2 \mathrm{ml}$ triethanolamine, $17.5 \mathrm{ml}$ of $10 \% \mathrm{w} / \mathrm{v}$ polyvinylalcohol stock solution and $4 \mathrm{ml}$ of $0.11 \% \mathrm{w} / \mathrm{v}$ of Methylene Blue stock solution. The photosensitive solution $(1 \mathrm{ml})$ was deposited on the levelled glass slide $\left(26 \times 76 \mathrm{~mm}^{2}\right)$ and dried for 24 hours in a dark room at ambient conditions $\left(\mathrm{T}=21 \pm 2{ }^{\circ} \mathrm{C}\right.$ and $\left.\mathrm{RH}=30-40 \%\right)$.

The transmission volume phase gratings were recorded using a standard two-beam setup (figure 1). 


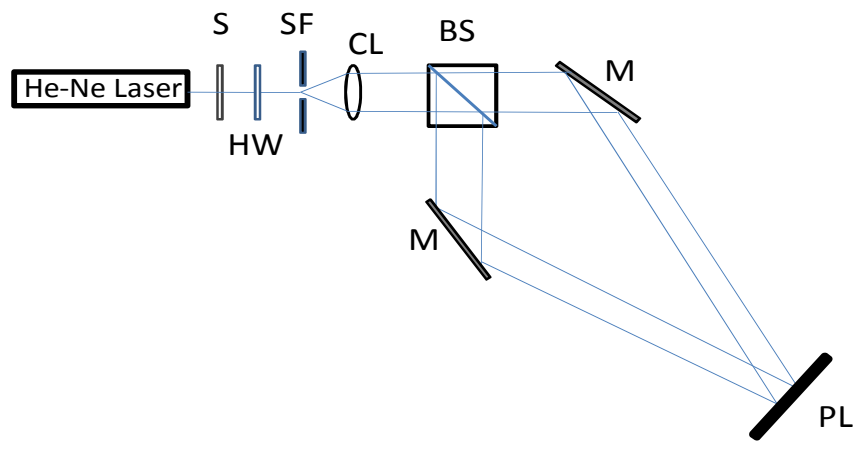

Figure 1. Recording set-up: S -electronic shutter; HW - half-wave plate; SF - spatial filter; CL collimator; BS - beam splitter; $\mathrm{M}$ - mirror; PL - photopolymer layer.

The photopolymer layers were exposed to two $633 \mathrm{~nm}$ beams obtained by splitting He-Ne laser beam. The total recording intensity was $5 \mathrm{~mW} / \mathrm{cm}^{2}$ and the recording time was $10 \mathrm{sec}$. Immediately after the recording the gratings were bleached under UV light for $60 \mathrm{~min}$ in order to polymerise all residual monomers. After bleaching, the absorption of the photopolymer layer at $633 \mathrm{~nm}$ was negligible, so $633 \mathrm{~nm}$ beam from He-Ne laser was employed as a probe beam for the Bragg selectivity curve measurements. To measure the Bragg selectivity curve, the sample was mounted on a rotation stage which was computer controlled via a motion controller (model Newport ESP300 with angular resolution of $0.1^{0}$ ). The Bragg selectivity curve measurement was performed by monitoring the firstorder diffracted beam intensity using an optical power meter (Newport Model 840) while the sample was rotated. The diffraction efficiency was defined as the ratio of the diffracted intensity and the intensity of the incident beam. LabVIEW software was used to plot the data in real time. The thickness of the dry layers was measured with a white light interferometric surface profiler MicroXAM S/N 8038. A controlled environment chamber with humidity and temperature control system (Electro-tech system, model 5503-11) was utilised to obtain different environmental conditions. The chamber was able to maintain the RH and temperature with accuracy of $\pm 1 \%$ and \pm 1 ${ }^{0} \mathrm{C}$, respectively.

The Bragg selectivity curve and thickness measurements had been carried out before and after humidity exposure. After humidity exposure, all the samples had been dried for 24 hours at ambient conditions $\left(\mathrm{T}=18 \pm 2{ }^{\circ} \mathrm{C}\right.$ and $\left.\mathrm{RH}=30-40 \%\right)$. The diffraction efficiency of the grating was estimated from the maximum of Bragg selectivity curve. The diffraction efficiency and the thickness, measured before and after humidity exposure, were assigned as $\eta(0), d(0)$ and $\eta(R H), d(R H)$, respectively.

\section{Results and Discussion}

\subsection{Diffraction efficiency change due to variation of relative humidity}

In order to characterise the influence of relative humidity on the diffraction efficiency, the gratings were placed in the humidity chamber and the diffraction efficiency was measured. For ease of comparison, normalized diffraction efficiency was used. The normalised diffraction efficiency was defined as the ratio of the diffraction efficiency at the current relative humidity to the diffraction 
efficiency at the start of the experiment, measured at relative humidity of $20 \%$. To minimize the inaccuracy caused by beam scattering due to water condensation on the photopolymer layer surface, intensities of transmitted $\left(I_{0}\right)$ and first-order diffracted $\left(I_{l}\right)$ beams were monitored. The readings were taken after $30 \mathrm{~min}$ of humidity exposure to allow the samples to equilibrate with the surrounding conditions and the diffraction efficiency in this particular experiment was defined as $I_{I} /\left(I_{I}+I_{0}\right)$. Figure 2 shows the dependence of normalized diffraction efficiency on relative humidity during humidity exposure at different temperatures.

As can be seen from figure 2 , at $20 \% \leq \mathrm{RH} \leq 70 \%$ the change in diffraction efficiency during humidity exposure does not depend on the temperature and follows the same trend for all three temperatures. However at $\mathrm{RH}=80 \%$ and $90 \%$ the normalized diffraction efficiency is different for different temperatures. As the temperature increases, the normalized diffraction efficiency drops further at the higher humidity.

It can also be observed in Fig.2 that for all three temperatures the normalised diffraction efficiency slightly increases in the relative humidity range $20-60 \%$. This is most probably due to swelling of the photopolymer layer as a result of absorption of moisture from the environment. The swelling is initially only in direction perpendicular to the glass substrate, as the good adhesion of the photopolymer layer to the glass substrate prevents dimensional change in direction along the surface. Thus effectively the thickness of the hologram is increased and the diffraction efficiency increases as well. Similar swelling/shrinkage occurring only in the vertical direction was previously observed in reflection $[8,12]$.

In addition it has been previously shown $[8,12]$, that the changes in diffraction efficiency of reflection gratings, recorded in acrylamide-based photopolymer layers, after exposure to $\mathrm{RH} \leq 80 \%$ are reversible. Since we aim to investigate irreversible changes, we focused our attention on high humidity $(\mathrm{RH}=80 \%$ and $90 \%)$.

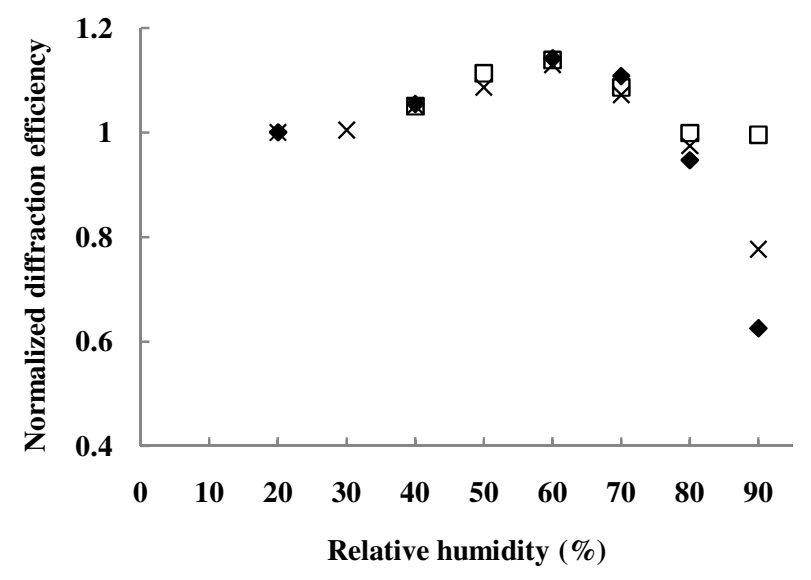

Figure 2. Normalized diffraction efficiency v/s relative humidity at temperature $8 \pm 1{ }^{0} \mathrm{C}(\square), 16 \pm 1$ ${ }^{0} \mathrm{C}(\mathrm{x}), 21 \pm 1{ }^{0} \mathrm{C}(\diamond)$.

\subsection{Diffraction efficiency change after exposure to $R H=80 \%$ and $90 \%$}

To investigate the reversibility of the diffraction efficiency changes observed during the exposure to high humidity, samples were exposed to high humidity at different temperatures for $60 \mathrm{~min}$ and left to recover at ambient conditions for 24 hours $\left(\mathrm{T}=18 \pm 2{ }^{\circ} \mathrm{C}\right.$ and $\left.\mathrm{RH}=30-40 \%\right)$. Normalized diffraction efficiency, calculated as the ratio of diffraction efficiency after humidity exposure $\eta(R H)$ 
to diffraction efficiency at the start of the experiment (before humidity exposure) $\eta(0)$, is presented in figure 3. We can conclude from the graph that, except for the lowest temperature of $8 \pm 1{ }^{0} \mathrm{C}$, exposure to high humidity results in an irreversible decrease of diffraction efficiency. The magnitude of the irreversible decrease also depends on temperature during humidity exposure and it is greatest at $\mathrm{T}=21 \pm 1{ }^{\circ} \mathrm{C}$ and $\mathrm{RH}=90 \%$.

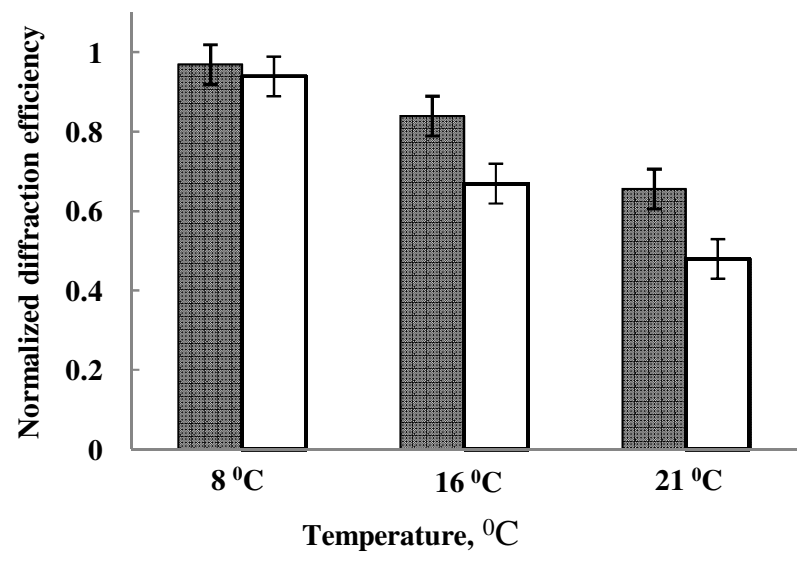

Figure 3. Normalized diffraction efficiency after exposure to high humidity: RH $=80 \%$ (घ) and $90 \%$ ( $\square$ ) at different temperatures.

\subsection{Diffraction efficiency change after exposure to $R H=90 \%$ : dependence on time of exposure}

In this section we investigate the effect of the duration of the humidity exposure on the observed change in diffraction efficiency. The time for which the grating has been exposed is found to have a significant effect on the diffraction efficiency after exposure. Figure 4 shows the normalized diffraction efficiency measured after exposure to high humidity for a set period of time at three different temperatures: $8 \pm 1{ }^{\circ} \mathrm{C}, 16 \pm 1{ }^{\circ} \mathrm{C}$ and $21 \pm 1{ }^{\circ} \mathrm{C}$. These samples were exposed to $\mathrm{RH}=90 \%$ for the specified amount of time and then were returned to normal humidity ( $\mathrm{RH}=30-40 \%$ ) before the measurements was taken. At $\mathrm{T}=8 \pm 1{ }^{\circ} \mathrm{C}$ a few percent reduction in diffraction efficiency is observed for samples exposed to up to 2 hours at high humidity. At $\mathrm{T}=16 \pm 1{ }^{\circ} \mathrm{C}$ and $21 \pm 1{ }^{\circ} \mathrm{C}$ normalized diffraction efficiency significantly declines during the first $40 \mathrm{~min}$ of exposure and then is almost constant. Fitting the experimental curves by single exponential decay, time constants were found to equal to $31 \mathrm{~min}, 22 \mathrm{~min}$ and $18 \mathrm{~min}$ at $8 \pm 1{ }^{\circ} \mathrm{C}, 16 \pm 1{ }^{\circ} \mathrm{C}$ and $21 \pm 1{ }^{\circ} \mathrm{C}$, respectively. Hence, the time constant is shorter, i.e. the process is faster, at higher temperature. From this result we can conclude that the magnitude of the irreversible decrease of diffraction efficiency depends on both humidity exposure time and temperature during humidity exposure. 


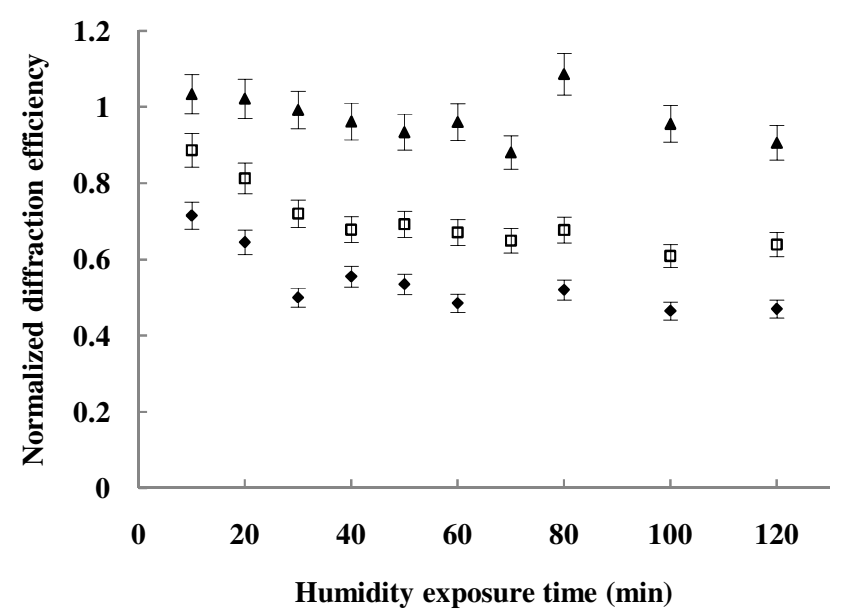

Figure 4. Normalized diffraction efficiency after exposure to $\mathrm{RH}=90 \%$ at $\mathrm{T}=8 \pm 1{ }^{0} \mathrm{C}(\boldsymbol{\Delta}), 16 \pm 1$ ${ }^{0} \mathrm{C}(\square), 21 \pm 1{ }^{0} \mathrm{C}(\downarrow)$ on humidity exposure time.

\subsection{Diffraction efficiency change after exposure to $R H=90 \%$ : dependence on temperature}

The temperature dependence of irreversible decrease of diffraction efficiency after exposure to $\mathrm{RH}=$ $90 \%$ has been investigated in more detail. The results are presented in figure 5. It can be seen that changes in normalized diffraction efficiency induced by exposure to $\mathrm{RH}=90 \%$ are fully reversible, when the temperature during the humidity exposure is kept below $9{ }^{\circ} \mathrm{C}$. At $9<\mathrm{T}<16{ }^{0} \mathrm{C}$ decrease of normalized diffraction efficiency is about few percents. In case the temperature exceeds $16{ }^{\circ} \mathrm{C}$ the changes become irreversible. Moreover, exposure to $\mathrm{RH}=90 \%$ at higher temperature leads to a larger drop in normalized diffraction efficiency. These results suggest that it might be that the structure changes in the holographic grating under the combined influence of high humidity and elevated temperature. It is well known that the freezing/melting temperature range of one of the ingredients of the photopolymer - triethanolamine is in the range of $17.9-21{ }^{0} \mathrm{C}$ [19]. Below this temperature range the created photonic structure is likely to be more stable and, consequently, the diffraction efficiency changes are reversible.

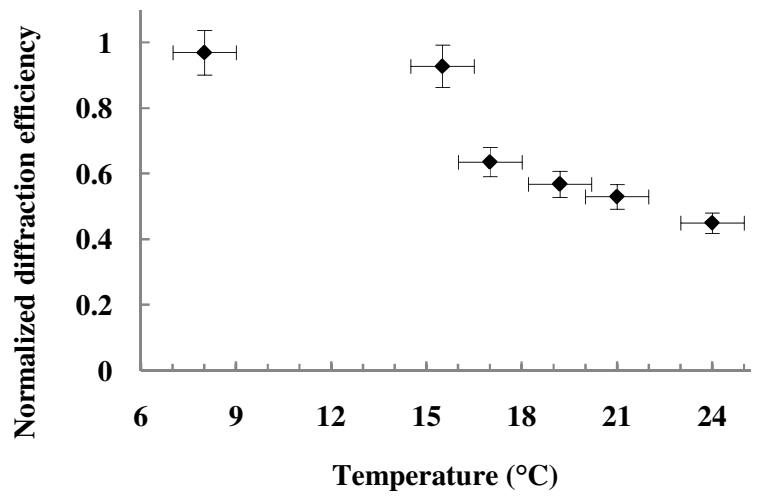

Figure 5. Temperature dependence of normalized diffraction efficiency after exposure to $\mathrm{RH}=90 \%$ for $60 \mathrm{~min}$. 
4.5. Change in grating thickness, Bragg angle and refractive index modulation 4.5.1. Change in grating thickness

According to the coupled wave theory [15], the change in diffraction efficiency implies a change in thickness and/or refractive index modulation in the recorded gratings. To better understand the processes behind the observed diffraction efficiency decrease, the change in thickness after exposure to $\mathrm{RH}=90 \%$ for different amount of time has been measured (figure 6). The graph shows normalized thickness as a function of time of exposure to high humidity. Normalized thickness was calculated as $d(R H) / d(0)$, where $d(R H)$ is the thickness after and $d(0)$ is the thickness before exposure to high humidity. It can be observed that irreversible decrease in thickness indeed takes place and is larger when the temperature is higher. It has also been visually observed that there is lateral dimensional change of the photopolymer layer as illustrated in figure 7. The thickness decrease and lateral stretch observed can be explained as follows: upon exposure to humidity the layer swells in all directions increasing the thickness and both lateral dimensions. Because the layer is typically centimetres long in the two lateral dimensions and only around 100 microns in the thickness dimension, the effect is much more noticeable in the layer width and length, which are seen to visibly increase and overhang the edge of the substrate by a few millimetres. In moderate humidity and/or lower temperatures the layer will recover its original dimensions fully when returned to the original conditions, but above certain temperatures and humidity levels, the layer is stretched beyond its capacity to recover and collapses to a lower thickness while remaining 'stretched' in the lateral dimensions.

Because of this lateral stretch the fringe spacing also changes irreversibly and Bragg's law (4) predicts a change in the Bragg angle. Experimentally this can be observed as a shift in the Bragg angular selectivity curve.

The Bragg angular selectivity curves were measured before and after exposure to high humidity for each transmission holographic grating.

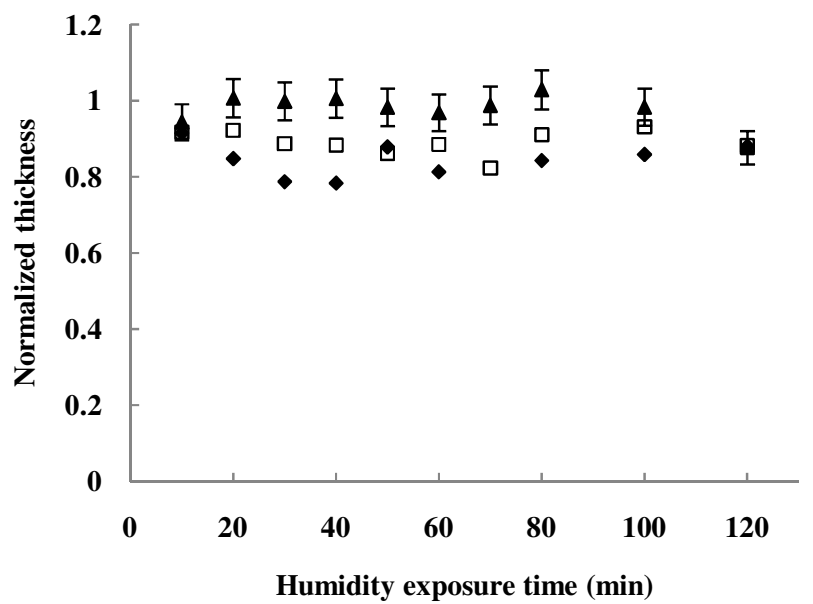

Figure 6. Normalized thickness after exposure to $\mathrm{RH}=90 \%$ at $\mathrm{T}=8 \pm 1{ }^{0} \mathrm{C}(\boldsymbol{\Delta}), 16 \pm 1{ }^{0} \mathrm{C}(\square), 21 \pm$ $1{ }^{0} \mathrm{C}(\bullet)$ on humidity exposure time. 
a)

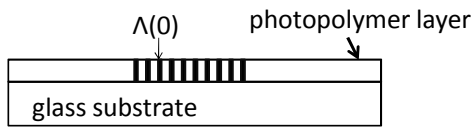

b)

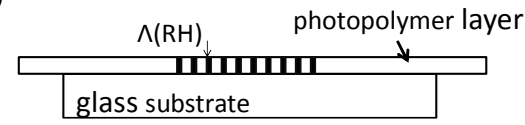

Figure 7. Schematic representation of the photopolymer layer before (a) and after (b) exposure to $\mathrm{RH}$ $=90 \%$ at $\mathrm{T}>15{ }^{\circ} \mathrm{C} \cdot \Lambda(0)$ and $\Lambda(R H)$ is the fringe spacing before and after humidity exposure, respectively.

\subsubsection{Shift in Bragg selectivity curve}

Figure 8 Illustrates the Bragg selectivity curve of a typical volume transmission grating measured before and after exposure to $\mathrm{RH}=90 \%$ at $\mathrm{T}=16 \pm 1{ }^{0} \mathrm{C}$ for $60 \mathrm{~min}$. By measuring the change in thickness it is possible to predict the change in the fringe spacing and thus calculate the expected Bragg angle shift using formula (4). The experimentally observed shift in Bragg angle is in a good agreement with Bragg angle shift calculated by (4) taking into account the change in thickness of the grating after humidity exposure as discussed below. The experimentally observed shift in Bragg angle confirms the irreversible change in fringe spacing of the transmission grating due to irreversible layer expansion in horizontal direction caused by exposure to high humidity. This irreversible expansion is most probably due stretching of the layer beyond the point of elastic deformation. As the photopolymer is a viscoelastic material, the irreversible layer expansion can be explained by decreasing layer viscosity due to water absorption and, hence, by decreasing its elasticity.

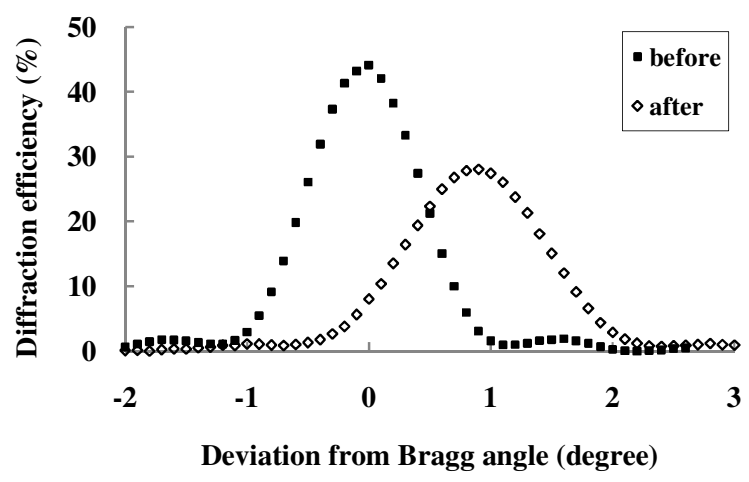

Figure 8. Bragg selectivity curve before and after exposure to $\mathrm{RH}=90 \%$ at $\mathrm{T}=16 \pm 1{ }^{\circ} \mathrm{C}$ for $60 \mathrm{~min}$. 


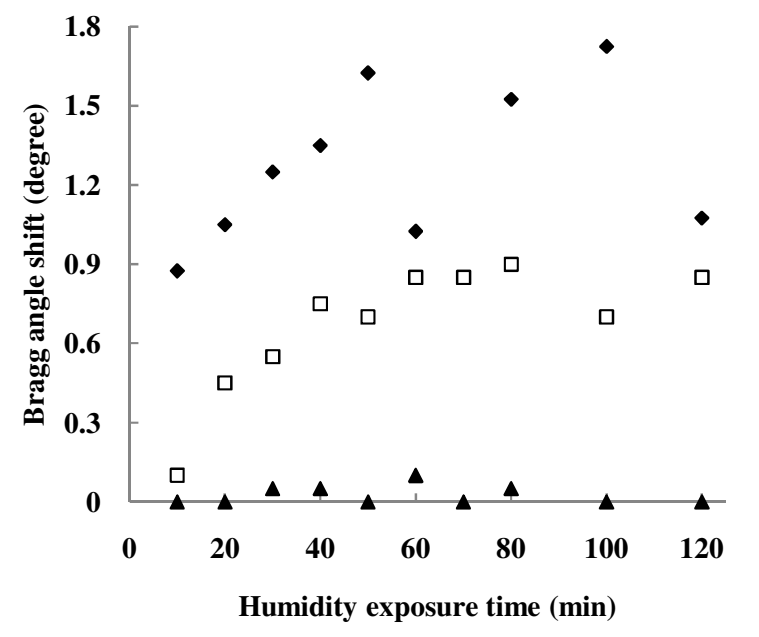

Figure 9. Bragg angle shift after exposure to $\mathrm{RH}=90 \%$ at $\mathrm{T}=8 \pm 1{ }^{0} \mathrm{C}(\boldsymbol{\Delta}), 16 \pm 1{ }^{0} \mathrm{C}(\square), 21 \pm 1{ }^{0} \mathrm{C}$ $(\downarrow)$ on humidity exposure time.

The shift in Bragg angle has been analysed for different exposure time at different temperatures (figure 9). It is clearly seen that the magnitude of Bragg angle shift (figure 9) follows the same trend as the thickness change (figure 6). At $\mathrm{T}=8 \pm 1{ }^{0} \mathrm{C}$ unchanged thickness is accompanied by a zero shift in Bragg angle. At higher temperatures shift in Bragg angle is bigger because of the bigger layer expansion. It is worth emphasizing again that the expected Bragg selectivity curve shift due to thickness change is in a good agreement with the measured value. So, for example, after exposure to $90 \%$ of RH for 100 min, the observed and calculated from the change in layer's dimensions shifts in Bragg angle for the three different temperatures are shown in table 1.

\begin{tabular}{|c|c|c|}
\hline Temperature, ${ }^{0} \mathrm{C}$ & $\begin{array}{c}\text { Measured Bragg angle } \\
\text { shift, }{ }^{0}\end{array}$ & $\begin{array}{c}\text { Calculated Bragg } \\
\text { angle shift, }\end{array}$ \\
\hline $8 \pm 1$ & 0 & 0 \\
\hline $16 \pm 1$ & 0.7 & 0.7 \\
\hline $21 \pm 1$ & 1.8 & 1.5 \\
\hline
\end{tabular}

Table 1 Comparison of the experimentally measured and calculated from the layer's thickness change Bragg angle shift at different temperatures.

Small discrepancy between observed and calculated shift in Bragg angle at $21 \pm 1{ }^{\circ} \mathrm{C}$ can be caused by thickness measurement error.

\subsubsection{Refractive index modulation}

It is important to understand whether other irreversible changes take place in the grating in addition to the dimensional deformation of the photopolymer layer. Such changes could be mass transport between the areas illuminated by dark and bright fringes during the exposure to high humidity. Since the density and the refractive index of the photopolymer is higher in the bright fringe areas, the decrease in the diffraction efficiency due to exposure to high humidity could be caused by effective decrease of the refractive index in the bright areas and/or effective increase of the refractive index in the dark fringe areas. A mass transport from bright to dark fringe areas could be the process causing 
the changes described above. Another explanation could be the different porosity of the grating's fringes (created during exposure to the bright and dark fringes of the recording interference pattern) and their different ability to retain water.

Using the thickness data and taking into account the shift in Bragg angle, we have estimated the refractive index modulation by formula (2). It has been found that the diffraction efficiency drop cannot be explained only by the thickness change and shift in Bragg angle. A small change in the refractive index modulation has also occurred.

Normalized refractive index modulation, defined as the ratio of $\Delta n(R H)$ - refractive index modulation after exposure to humidity and $\Delta n(0)$ - refractive index modulation before humidity exposure, is used to analyse the change in refractive index modulation. As can be seen from figure 10, there is no change in normalized refractive index modulation caused by exposure to $\mathrm{RH}=90 \%$ at $\mathrm{T}=8 \pm 1{ }^{\circ} \mathrm{C}$.

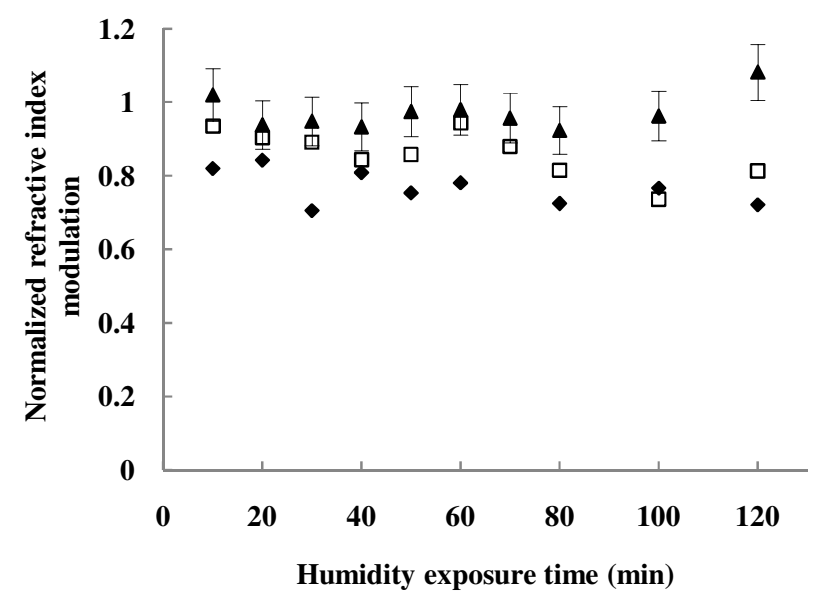

Figure 10. Normalized refractive index modulation after exposure to $\mathrm{RH}=90 \%$ at $\mathrm{T}=8 \pm 1{ }^{0} \mathrm{C}(\boldsymbol{\Delta})$, $16 \pm 1{ }^{0} \mathrm{C}(\square), 21 \pm 1{ }^{0} \mathrm{C}(\diamond)$ on humidity exposure time.

This result is in a good agreement with unchanged normalized diffraction efficiency (figure 4). Exposure to high humidity at $\mathrm{T}=16 \pm 1{ }^{\circ} \mathrm{C}$ and $21 \pm 1{ }^{\circ} \mathrm{C}$ results in decrease of refractive index modulation and, consequently, normalized diffraction efficiency is also reduced (figure 4).

\section{Conclusions}

Investigation of the volume transmission grating properties after exposure to high humidity has been carried out. Both diffraction efficiency and Bragg selectivity are observed to change irreversibly at higher temperature, and full reversibility was confirmed for lower temperatures. It has also been established that the shift in Bragg selectivity curve can be explained by the irreversible dimensional change in layers. Irreversible changes in diffraction efficiency, however, are caused by this change in thickness and a change in the refractive index modulation implying some diffusion processes occur more freely as the layer takes on moisture. The magnitude of the irreversible change highly depends on humidity level, temperature during the humidity exposure and on humidity exposure time. If the gratings are kept below $9{ }^{\circ} \mathrm{C}$, the changes in diffraction efficiency are fully reversible.

This sensitivity of transmission gratings, recorded in acrylamide-based photopolymer layers, to high humidity ( $\mathrm{RH} \geq 80 \%$ ) can be utilized for the development of irreversible humidity holographic sensors, but also can limit the application of this material when non-sensitive to the environment material is needed. The fuller understanding of the processes involved limits past which irreversible 
change occurs and quantification of those changes helps in the design of sensors and also in the development of new version of acrylamide based photopolymer which is less sensitive to humidity. Further work in this direction will be published shortly.

\section{Acknowledgments}

The authors would like to acknowledge the Irish Research Council Embark Initiative for financial support and also thank the FOCAS Research Institute for providing research facilities.

\section{References}

1. Martin S, Feely C A and Toal V 1997 Holographic recording characteristics of an acrylamidebased photopolymer Appl. Optics 36 5757-68

2. Guntaka S, Toal V and Martin S 2002 Holographically recorded photopolymer diffractive optical element for holographic and electronic speckle-pattern interferometry Appl. Optics $\mathbf{4 1}$ 7475-79

3. Yao H, Huang M, Chen Z, Hou L and Gan F 2002 Optimization of two-monomer-based photopolymer used for holographic recording Materials Letters 56 3-8

4. Blaya S, Carretero L, Madrigal R F and Fimia A 2003 Optimization of a photopolymerizable holographic recording material based on polyvinylalcohol using angular responses Optical Materials 23 529-38

5. Huang M, Wang S, Wang A, Gong Q and Gan F 2005 A wideband sensitive holographic photopolymer Chinese Optics Letters 3 268-70

6. Liu S, Gleeson M and Sheridan T 2009 Analysis of the photoabsorptive behaviour of two different photosensitizers in a photopolymer material J. Opt. Soc .Am. B 26 528-36

7. Zhu J, Wang G, Hao Y, Xie B and Cheng A 2010 Highly sensitive and spatially resolved polyvinylalcohol/acrylamide photopolymer for real-time holographic applications Optics Express 18 18106-12

8. Naydenova I, Jallapuram R, Toal V and Martin S 2008 A visual indication of environmental humidity using a colour changing hologram recorded in a self-developing photopolymer Appl. Phys. Lett 92031109

9. Fernandez E, Garcia C, Pascual I, Ortuno M, Gallego S and Belendez A 2006 Optimization of a thick polyvinyl alcohol-acrylamide photopolymer for data storage using a combination of angular and peristrophic holographic multiplexing Applied Optics 45 7661-66

10. Gallego S, Marquez A, Marini S, Fernandez E, Ortuno M and Pascual I 2009 In dark analysis of PVA/AA materials at very low spatial frequencies: phase modulation evolution and diffusion estimation Optics Express 17 18279-291

11. Gong Q, Wang S, Huang M and Gan F 2005 A humidity-resistant highly sensitive holographic photopolymerizable dry film Materials Letters 59 2969-72

12. Naydenova I, Jallapuram R, Toal V and Martin S 2009 Characterization of the humidity and temperature responses of a reflection hologram recorded in acrylamide-based photopolymer Sensors and Actuators B: Chemical 139 35-8

13. Leite E, Babeva T, Ng E, Toal V, Mintova S and Naydenova I 2010 Optical Properties of Photopolymer Layers Doped with Aluminophosphate Nanocrystals Journal of Physical Chemistry C 114 16767-75

14. Phariseau P 1956 On the diffraction of light by progressive supersonic waves Proc. Ind. Acad. Sci. 44A $165-70$

15. Kogelnic H 1969 Coupled wave theory for thick hologram gratings Bell Syst. Tech. J. 48 290947

16. Martin S, Leclere P, Renotte Y, Toal V and Lion Y 1994 Characterization of an acrylamidebased dry photopolymer holographic recording material Opt. Eng. 33 3942-6

17. Naydenova I, Martin S, Jallapuram R, Howard R and Toal V 2004 Investigations of the diffusion processes in self-processing acrylamide-based photopolymer system Applied Optics $\mathbf{4 3}$ $2900-5$ 
18. Babeva T, Naydenova I, Mackey D, Martin S and Toal V 2010 Two-way diffusion model for short-exposure holographic grating formation in acrylamide based photopolymer JOSA B 27 197-203

19. Material Safety Data Sheet-16303, http://www.sigma-aldrich.com/ 